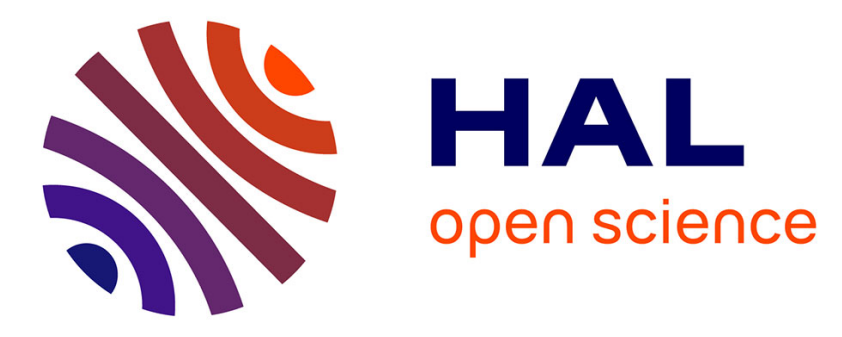

\title{
Kinetics and Energetics of Ge Condensation in SiGe Oxidation
}

Thomas David, Abdelmalek Benkouider, Jean-Noël Aqua, Martiane Cabie, Luc Favre, Thomas Neisius, Marco Abbarchi, Meher Naffouti, Antoine Ronda, Kailang Liu, et al.

\section{To cite this version:}

Thomas David, Abdelmalek Benkouider, Jean-Noël Aqua, Martiane Cabie, Luc Favre, et al.. Kinetics and Energetics of Ge Condensation in SiGe Oxidation. Journal of Physical Chemistry C, 2015, 119 (43), pp.24606 - 24613. 10.1021/acs.jpcc.5b07062 . hal-01473115

\section{HAL Id: hal-01473115 https: / hal.sorbonne-universite.fr/hal-01473115}

Submitted on 21 Feb 2017

HAL is a multi-disciplinary open access archive for the deposit and dissemination of scientific research documents, whether they are published or not. The documents may come from teaching and research institutions in France or abroad, or from public or private research centers.
L'archive ouverte pluridisciplinaire HAL, est destinée au dépôt et à la diffusion de documents scientifiques de niveau recherche, publiés ou non, émanant des établissements d'enseignement et de recherche français ou étrangers, des laboratoires publics ou privés. 


\title{
Kinetics and Energetics of Ge Condensation in SiGe Oxidation
}

\author{
Thomas David, ${ }^{* \dagger}$ Abdelmalek Benkouider, ${ }^{\dagger}$ Jean-Noël Aqua,${ }^{\ddagger}$ Martiane Cabie, \\ Luc Favre, ${ }^{\S}$ Thomas Neisius,,${ }^{\S}$ Marco Abbarchi, ${ }^{\dagger}$ Meher Naffouti, ${ }^{\dagger}$ Antoine \\ Ronda, $^{\dagger}$ Kailang Liu, ${ }^{\dagger}$ and Isabelle Berbezier ${ }^{\dagger}$ \\ $\dagger$ †ix-Marseille Univ. - CNRS, IM2NP, Faculté des Sciences de Jérôme, F-13397 Marseille \\ France. \\ $\ddagger I N S P$, Boite 840; 4 pl. Jussieu; 75005 Paris. \\ ๑Aix-Marseille Univ, CP2M, Faculté des Sciences de Jérôme, F-13397 Marseille France.

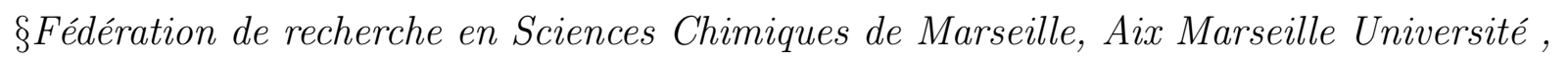 \\ Campus St Jérôme, 13397 Marseille cedex 20, France. \\ E-mail: thomas.david@im2np.fr \\ Phone: + 33 (0) 491289163
}




\begin{abstract}
The fabrication of ultra-thin Ge-rich SiGe body on Silicon On Insulator (SOI) is highly challenging for the next generation of fully depleted CMOS devices that will be implemented in the near future. Ge-rich layers (GRLs) could be fabricated using a Ge enrichment process which takes place during dry thermal oxidation of SiGe thin films. While several studies make use of the GRL for many applications, the basic mechanism at work during the enrichment process is still unclear. In this study, we address the mechanism of formation of GRL and we determine the major driving forces of the enrichment process. We highlight the particular role played by the $\mathrm{Si}_{0.5} \mathrm{Ge}_{0.5}$ which is stabilized for various experimental conditions and strain levels. A systematic study demonstrates that the $50 \%$ Ge content is stabilized by a self-limited interdiffusion process regulated by the entropic term of the formation energy which is minimum at $\mathrm{Si}_{0.5} \mathrm{Ge}_{0.5}$ at the expense of the elasticity driven interdiffusion. The process developed provides an easy and efficient way to produce planar GRLs free of dislocations with abrupt GRL/Si interfaces and tunable thickness. These GRLs could be fashioned for the heterogeneous integration of various systems on SOI.
\end{abstract}

\title{
Introduction
}

The stability of SiGe layers and the mechanisms of ultra-thin film growth on silicon on insulator (SOI) are issues of crucial importance for the fabrication of the next generation fully depleted CMOS devices. ${ }^{1-5}$ SiGe is a commonly used material in CMOS technology because of its strain-induced transport and optical properties. ${ }^{6}$ The Ge condensation process has been reported as a relevant way for fabricating ultra-thin Ge-rich SiGe layers (GRLs) on SOI with a controlled thickness and composition. ${ }^{7-13}$ The process takes place during dry thermal oxidation of $\mathrm{SiGe}$ which consumes $\mathrm{Si}$ atoms to form a $\mathrm{SiO}_{2}$ layer on top of the $\mathrm{SiGe}$. In parallel, the Ge atoms pile up at this new $\mathrm{SiGe} / \mathrm{SiO}_{2}$ interface, forming the GRL without loss of Ge until the silicon is fully oxidized. ${ }^{14}$ The enrichment mechanism has been explained 
by the much lower formation energy of $\mathrm{SiO}_{2}$ than $\mathrm{GeO}_{2}\left(\mathrm{E}_{\mathrm{SiO}_{2}}=-8.2 \mathrm{eV}\right.$ and $\mathrm{E}_{\mathrm{GeO}_{2}}=-4.7 \mathrm{eV}$ respectively) which highly favors the formation of $\mathrm{SiO}_{2}$ against $\mathrm{GeO}_{2} \cdot{ }^{15}$

A quite different mechanism was observed during low temperature plasma-assisted oxidation. The films generated by these processes have been found to be mixtures of stoichiometric $\mathrm{SiO}_{2}$ and $\mathrm{GeO}_{2}$ and the kinetics of oxidation depends on the Ge concentration of the alloy. ${ }^{16}$ At the opposite, during dry thermal oxidation the kinetics is almost independent of the Ge content and the oxide contains only $\mathrm{Si}$ atoms. ${ }^{17} \mathrm{~A}$ crucial parameter which dramatically changes the formation of GRLs is the oxidation temperature. It was reported that the temperature may be set such that the Ge content at the oxide interface is increased, kept static, or decreased. ${ }^{18}$ Two different temperature regimes of oxidation can clearly be distinguished depending on the application foreseen.

The high temperature regime is commonly chosen (without exceeding the melting point of the SiGe) to obtain an homogeneous germanium concentration. In these conditions, there is no formation of GRL since the Ge in excess at the SiGe/oxide interface is immediately redistributed inside the SiGe layer due to the large Ge/SiGe interdiffusion coefficient at these temperatures. The fabrication of perfectly homogeneous SiGe layers at high temperature was reported. ${ }^{19-21}$ In similar conditions, the formation of pure germanium on insulator has also been reported ${ }^{22}$ while in other studies, on the contrary, a saturation concentration at $\mathrm{x}=0.5$ was mentioned. It was suggested that above this concentration, both silicon and germanium are consumed during oxidation inhibiting the enrichment process and making thereby the previously mentioned formation of pure germanium on insulator impossible. ${ }^{22}$

At the opposite, the low temperature regime is commonly used for the fabrication of GRLs with abrupt interfaces. ${ }^{19}$ In this regime, the diffusion of Ge atoms inside the SiGe remains quite low while the oxidation rate is higher due to the larger diffusion coefficient of oxygen in the $\mathrm{SiO}_{2}$ (required for the oxidation). ${ }^{23} \mathrm{~A}$ major problem in this regime is the large strain energy stored in the heteroepitaxial system, which could be easily relaxed by the nucleation of extended defects. The concentration variations and resulting strain must thus 
be very precisely controlled to avoid any plastic relaxation. Several processes were suggested to suppress the nucleation of dislocations. ${ }^{24,25}$

Many studies rely on the effect of strain at the $\mathrm{SiO}_{2} / \mathrm{Si}$ interface to assess the segregation of $\mathrm{Ge}$ and its self-limitation. It is suggested that the strain-enhanced diffusion favors the diffusion of Ge towards the Si substrate and increases the homogenization of the SiGe layer, thus reducing the strain energy of the system.

Given the contradictory results reported in the literature, a detailed understanding of the kinetics of SiGe oxidation and more precisely the Ge pile-up mechanism is required to optimize the growth of Ge rich SiGe layers on insulator and overcome the actual technological barriers that limit the fabrication of MOSFET devices for the next generation of sub-28nm nodes. The two major questions addressed here concern first the kinetic and energetic control of the GRL formation and second the stabilization of the $50 \%$ concentration. In this context we use two types of samples with different strain levels and we investigate systematically the kinetics of oxidation and the physico-chemical mechanism of GRLs formation.

We demonstrate the formation of GRLs with a constant concentration of $50 \%$ which remain stable in different experimental conditions (oxidation time and temperature, strain state). We show that neither the strain, nor the minimization of the free energy can explain the stability of this concentration. We therefore suggest that an additional driving force is at the origin of the stabilization of this GRL concentration.

\section{Experimental details}

\section{Fabrication process}

Two different initial structures were used to study the oxidation and the condensation mechanisms (figure 1). The first structures consist of a $1 \mu$ m thick $\mathrm{Si}_{0.8} \mathrm{Ge}_{0.2}$ relaxed layer on a graded SiGe buffer layer epitaxially grown on silicon. They are used in the first series of experiments focusing on the kinetics of oxidation and condensation. 


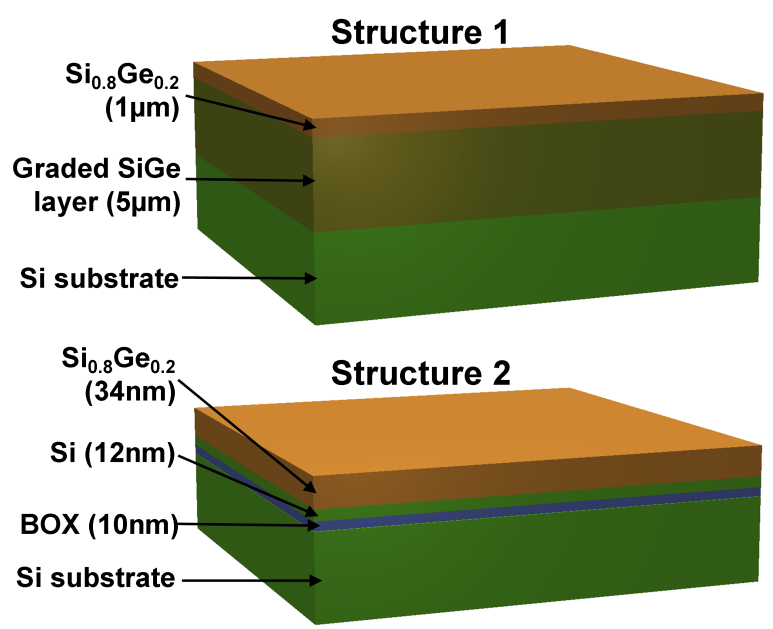

Figure 1: The two different structures before oxidation. $\mathrm{Si}_{0.8} \mathrm{Ge}_{0.2}$ is relaxed in structure 1 whereas it is epitaxially grown on silicon in structure 2 .

The second structures consist of a $34 \mathrm{~nm}$ thick layer of $\mathrm{Si}_{0.8} \mathrm{Ge}_{0.2}$ deposited by molecular beam epitaxy (MBE) at $450{ }^{\circ} \mathrm{C}$ on an ultra-thin silicon on insulator (UTSOI). The thickness of the top layer and buried oxide (BOX) are $12 \mathrm{~nm}$ and $10 \mathrm{~nm}$ respectively. These structures are used to study the condensation mechanism at low temperature and to determine the microstructural and chemical evolution of the GRLs with oxidation time.

Using these two different initial structures allows the condensation mechanism to be decoupled from the strain effect since it is studied on both a relaxed (first thick structure) and strained (second thin structure) initial SiGe layer. Furthermore, the second structure only contains a limited quantity of silicon atoms (above the BOX), and thus allows the study of the oxidation when there is no more reservoir of pure silicon.

The MBE growth chamber is a RIBER MBE32. The oxidations are performed in a rapid thermal oxidation (RTO) furnace JIPELEC. Oxidation were performed at temperatures between 700 and $950{ }^{\circ} \mathrm{C}$; duration was varied between $1 \mathrm{~h} 30$ and $15 \mathrm{~h}$ by steps of $30 \mathrm{~min}$ in accordance with the RTO technical specifications.

The germanium condensation step illustrated in figure 2 is taking place during dry thermal oxidation (in $\mathrm{O}_{2}$ atmosphere) of the initial SiGe structures. During oxidation there are two competing processes: (1) the incorporation of oxygen atoms inside the SiGe layer prefer- 

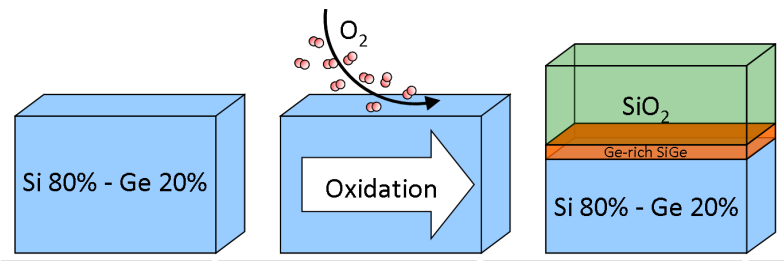

Figure 2: Oxidation of SiGe and formation of a Ge-rich layer by condensation of the germanium at the interface.

entially within SiâĂŞSi bonds. The process that requires the lowest activation energy is the jump from one Si-Si line to a neighboring Si-Si line in such a way that only one Si-O bond is broken; (2) the diffusion of Ge towards the SiGe buffer layer. During this process, the Ge atoms (which remain non-bonded to oxygen atoms) are repulsed below the new $\mathrm{SiO}_{2} / \mathrm{SiGe}$ interface, forming a Ge-rich SiGe layer (GRL). Keeping a low temperature (below $850{ }^{\circ} \mathrm{C}$ ) limits the diffusion of Ge atoms inside the SiGe, reducing the extension of the GRL while the rate of incorporation of oxygen atoms in the SiGe layer remains quite high. At higher temperatures, the diffusion of Ge in $\mathrm{SiGe}$ (or $\mathrm{Si}$ ) becomes easier. When the initial SiGe nominal layer is quite thin (for instance when SiGe is epitaxially deposited on SOI), the process results in the disappearance of the GRL and the homogenization of the Ge concentration in the whole SiGe layer.

\section{Microscopy}

Details on the structure and chemical composition are obtained using a FEI Tecnai G2, and a FEI Titan 80-300 with Cs corrector in TEM and STEM modes. EDS analyses and line profiles are performed with the FEI Titan 80-300 with Cs corrector using a probe size of approximately $1 \mathrm{~nm}$ or the FEI Tecnai G2 using a probe size of approximately $4 \mathrm{~nm}$. The $\mathrm{k}$ factors of $\mathrm{Si}, \mathrm{Ge}$ and $\mathrm{O}$ are measured on reference samples and allow the determination of absolute concentrations. Cross section samples are prepared using a dual-beam FIB HELIOS 600 nanolab or tripod polishing followed by PIPS thinning. 


\section{Results and analysis}

Two series of experiments were carried out to study the effect of the kinetics of oxidation and the basic mechanism of GRL formation and stabilisation. The aim is to determine first the driving forces of GRL formation when there is no epitaxial strain (relaxed nominal $\mathrm{Si}_{0.8} \mathrm{Ge}_{0.2}$ ) and second the morphological, microstructural and chemical evolution of the GRL during oxidation.

\section{Kinetics of SiGe oxidation and GRL formation}

A first series of experiments is performed to study the influence of temperature on the oxidation/condensation processes. In these experiments, no strain is present in the initial structure, which consists of a fully relaxed $(1 \mu \mathrm{m})$ thick $\mathrm{Si}_{0.8} \mathrm{Ge}_{0.2}$ layer, as previously described. The samples are oxidized for $90 \mathrm{~min}$ at various temperatures ranging from $700{ }^{\circ} \mathrm{C}$ to $950{ }^{\circ} \mathrm{C}$.

TEM cross-section images (Figure 3a) after oxidation evidence a dark layer at the $\mathrm{SiO}_{2} / \mathrm{Si}_{0.8} \mathrm{Ge}_{0.2}$ interface. The layer thickness increases with the temperature of oxidation (Tox). The GRLs remain epitaxially strained, perfectly planar and free of dislocation from $700{ }^{\circ} \mathrm{C}$ to $950{ }^{\circ} \mathrm{C}$. For sake of clarity, the TEM image of the sample obtained at $\left(950{ }^{\circ} \mathrm{C}\right)$ is not presented here to preserve a similar scale for all the images. The germanium concentration profiles were measured by STEM-EDS semi-quantitative analysis on the TEM cross section samples (Figure 3b). Two different regimes can be distinguished. At low temperatures, the thin GRLs exhibit an abrupt interface with the rest of the initial $\mathrm{Si}_{0.8} \mathrm{Ge}_{0.2}$ layer underneath. A constant concentration of $50 \%$ is measured for the samples oxidized up to Tox $\leq 900{ }^{\circ} \mathrm{C}$. At higher temperatures (Tox $\geq 900{ }^{\circ} \mathrm{C}$ ) a significant broadening of the interface is clearly visible accompanied by a progressive decrease of the Ge content. The latter behavior is consistent with the diffusion of $\mathrm{Ge}$ in the nominal $\mathrm{Si}_{0.8} \mathrm{Ge}_{0.2}$ layer. The interface broadening observed on the TEM images matches well previous results of the literature ${ }^{23}$ which exhibited the 

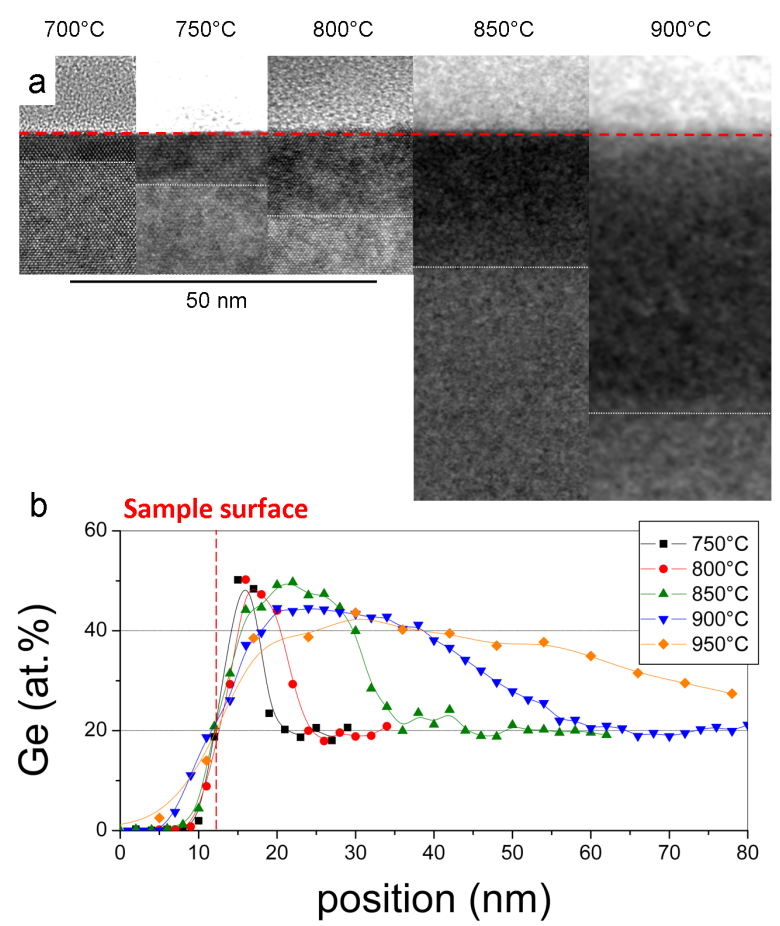

Figure 3: (a) HRTEM cross-section images of the $\mathrm{Si}_{0.8} \mathrm{Ge}_{0.2} / \mathrm{GRL} / \mathrm{SiO} 2$ system after oxidation at Tox $=700,750,800,850,900^{\circ} \mathrm{C}$. All the images have the same scale. (b)STEM-EDS line profiles of Ge concentration along the depth of the GRL for the different temperatures $\left(750-950^{\circ} \mathrm{C}\right)$.

homogenization of the SiGe concentration.

On Figure 4 are drawn the evolution of the square of the thickness normalized by the time of both the oxide and the GRL as a function of the inverse of the temperature with logarithmic scale. The dashed lines give the best fitting curves based on an Arrhenius expression $\left(y=a e^{-b x}\right)$ and allow to deduce an activation energy: $E_{a}=-k_{B} \times b$, with $k_{B}=8 \cdot 617 \cdot 10^{-5} \mathrm{eV} \cdot \mathrm{K}^{-1}$. The deduced activation energy of oxidation is about $2.1 \mathrm{eV} \pm 0.3$ $\mathrm{eV}$, which is between the activation energy of oxygen diffusion in $\mathrm{Si}$ and in Ge, approximately $2.5 \mathrm{eV}$ in $\mathrm{Si}^{26,27}$ and approximately $2 \mathrm{eV}$ in $\mathrm{Ge}^{28}$

An activation energy of approximately $2.56 \mathrm{eV}$ for the GRL growth is deduced. Given the measurement errors, this value can be considered close to the one of oxidation and suggests that the GRL formation is controlled (and limited) by the diffusion of oxygen atoms inside the SiGe layer. This result also demonstrates that Ge is not oxidized during the process in 


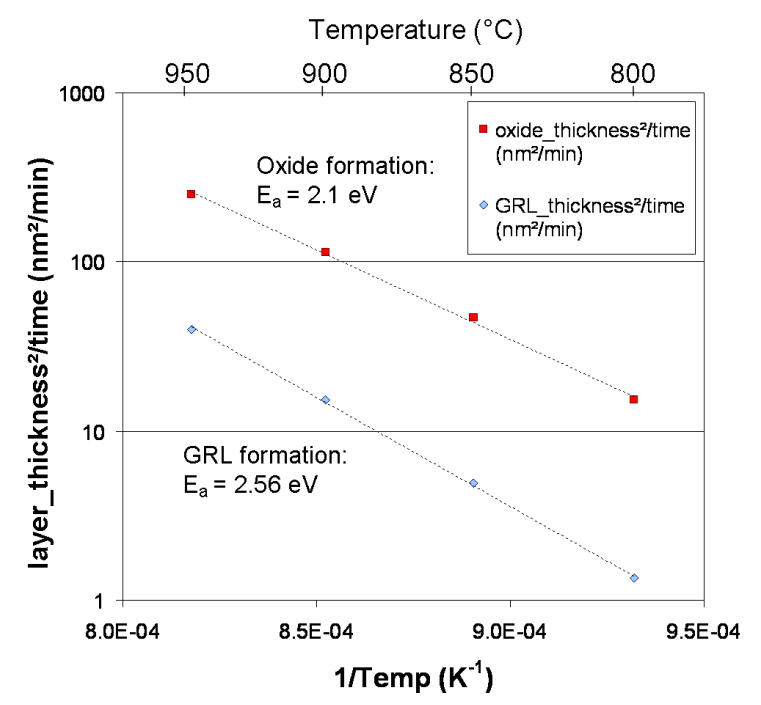

Figure 4: Oxide (top curve) and GRL (bottom curve) formation rates as a function of the inverse of the oxidation temperature. The slopes of fitting curves allow the determination of activation energies.

agreement with the EDS measurements. In addition, the measured value is also in the same range as the activation energy of the Ge diffusion in $\mathrm{Si}_{0.5} \mathrm{Ge}_{0.5}$.

From these results we can deduce that two different regimes are observed: at low temperature, the high kinetic barrier of diffusion of $\mathrm{Ge}$ in $\mathrm{Si}_{0.8} \mathrm{Ge}_{0.2}$ inhibits the diffusion. In this regime, the GRL layer grows and maintains a $50 \%$ pseudo-equilibrium concentration. At high temperature, the diffusion barrier is lower and Ge diffuses into $\mathrm{Si}_{0.8} \mathrm{Ge}_{0.2}$. The $\mathrm{Si}_{0.8} \mathrm{Ge}_{0.2} / \mathrm{Si}_{0.5} \mathrm{Ge}_{0.5}$ interface broadens and the Ge concentration of the GRL decreases. In this second regime, the diffusion towards the low concentration nominal SiGe layer induces a rapid redistribution of Ge atoms which homogenizes the SiGe layer concentration in agreement with previous results. ${ }^{23}$

To clarify the driving forces of stabilization of the GRL with $\mathrm{x}=0.5$ at low temperature, a second batch of experiments with a different initial structure was performed. 


\section{Evolution of GRLs with time}

In this section we focus on the oxidation of an heterostructure which consists of $34 \mathrm{~nm}$ thick $\mathrm{Si}_{0.8} \mathrm{Ge}_{0.2}$ layer epitaxially grown on a $12 \mathrm{~nm}$ UTSOI substrate (Figure 5).

The oxidation takes place at the GRL/oxide interface throughout the oxidation process, in parallel, the GRL grows in the direction of the substrate. The graph of figure 5a presents EDS line profiles of the germanium concentration along the depth for different oxidation duration (at $750{ }^{\circ} \mathrm{C}$ ). The precise distance to the $\mathrm{Si}(001)$ substrate/BOX interface is determined using oxygen and silicon profiles recorded at the same time (not shown for clarity). The initial structure and its evolution with time is drawn below the graph for reference (figure 5b). In figure 5c, dark-field STEM cross-section images corresponding to each oxidation duration are displayed on the same scale.

After 1.5 hours of oxidation, we observe the formation of a $5 \mathrm{~nm}$ thick GRL while part of the $\mathrm{Si}_{0.8} \mathrm{Ge}_{0.2}$ layer is still present. The profile obtained here matches well the situation already observed in the first part when oxidizing the fully relaxed $\mathrm{Si}_{0.8} \mathrm{Ge}_{0.2}$ susbtrate at $750 \hat{A} \check{r} \mathrm{C}$. Over time, the thickness of this GRL increases and the thickness of $\mathrm{Si}_{0.8} \mathrm{Ge}_{0.2}$ decreases. After 6 hours, all the $\mathrm{Si}_{0.8} \mathrm{Ge}_{0.2}$ is transformed into a $10 \mathrm{~nm}$ thick $\mathrm{Si}_{0.5} \mathrm{Ge}_{0.5}$ GRL. After 13 hours, this $10 \mathrm{~nm}$ thick GRL maintained the same thickness and the same concentration, while the entire underlying silicon layer has been oxidized. It is only after 14 hours that the Ge concentration in this layer increases (and reaches $\mathrm{Si}_{0.3} \mathrm{Ge}_{0.7}$ ) and its thickness decreases, until 15 hours which provides a $5 \mathrm{~nm}$ thick pure Ge layer.

Figure 6 shows HRTEM and high resolution STEM-HAADF images of the structure obtained after 8 hours. The thickness of the GRL and UTSOI correspond perfectly with the EDS profiles. One can note the total absence of extended defects and the very sharp variation of Ge concentration at the interface.

It is also important to note that we did not detect germanium in the top oxide layer (by EDS). This suggests that mainly silicon oxide is formed and that most of the germanium present in the initial $34 \mathrm{~nm}$ thick $\mathrm{Si}_{0.8} \mathrm{Ge}_{0.2}$ layer is pushed in the $10 \mathrm{~nm}$ thick $\mathrm{Si}_{0.5} \mathrm{Ge}_{0.5}$ layer. 
However, the evalutation of the total quantity of Ge from the thicknesses and concentrations of the layers at various times of the oxidation shows that part of germanium has been lost during the process (less than $10 \%$ ) by oxidation (formation of $\mathrm{GeO}_{2}$ ). A protective cap of a few monolayers of silicon is sometimes deposited on top of the SiGe to avoid the latter effect. ${ }^{22,29,30}$

Three different GRL growth steps can be distinguished: the first step $\left(t_{1}=6 \mathrm{~h}\right)$ is the complete oxidation of the $\mathrm{Si}_{0.8} \mathrm{Ge}_{0.2}$ layer. The oxidation and the GRL thickness increase with the square root of time which is representative of a diffusion limited mechanism. During this stage, the whole $\mathrm{Si}_{0.8} \mathrm{Ge}_{0.2}$ layer is transformed into $\mathrm{Si}_{0.5} \mathrm{Ge}_{0.5}$. We observe the thickening of the GRL at the expense of the $\mathrm{Si}_{0.8} \mathrm{Ge}_{0.2}$ layer and its displacment in the direction of the UTSOI substrate.

The second step is the oxidation of the Si layer of the initial UTSOI $\left(t_{2}=7 \mathrm{~h}\right)$. During this step, only the UTSOI is oxidized, the GRL thickness stays constant and it is pushed towards the buried oxide (BOX). The concentration of the GRL remains constant at $\mathrm{x}=0.5$. This step finishes when the initial UTSOI is fully oxidized.

The third step $\left(t_{3}=2 \mathrm{~h}\right)$ corresponds to the oxidation of the GRL. During this step, Si atoms present in the $\mathrm{Si}_{0.5} \mathrm{Ge}_{0.5}$ GRL are oxidized and the Ge concentration of the GRL increases while its thickness decreases. This step produces an enrichment of the GRL at concentrations higher than $\mathrm{x}=0.5$ and up to $\mathrm{x}=1$ (figure $5 \mathrm{c}$ ).

\section{Discussion}

A very interesting observation is the constant concentration at $\mathrm{x}=0.5$ of the GRL whatever the structure configuration and its strain level are. Indeed, in the first section there was no strain in the initial structure (which is a SiGe relaxed buffer layer), while in the second section the initial system ( $\mathrm{SiGe/UTSOI)}$ is fully strained and in addition the epitaxial compressive bi-dimensionnal strain evolves during time with the thickness ratio of the SiGe/UTSOI layers.

We have demonstrated that at the begining of the oxidation process, a thin $\mathrm{Si}_{0.5} \mathrm{Ge}_{0.5}$ 
layer immediately forms at the $\mathrm{Si}_{0.8} \mathrm{Ge}_{0.2} / \mathrm{SiO}_{2}$ interface.

Then, depending on the temperature, two different condensation regimes should be distinguished: at low temperature, there is no diffusion of $\mathrm{Ge}$ in the $\mathrm{Si}_{0.8} \mathrm{Ge}_{0.2}$ layer and the $\mathrm{x}=0.5$ concentration doesn't change during the full process. At high temperature, Ge diffuses in $\mathrm{Si}_{0.8} \mathrm{Ge}_{0.2}$. The consequences are the broadening of the $\mathrm{Si}_{0.5} \mathrm{Ge}_{0.5} / \mathrm{Si}_{0.8} \mathrm{Ge}_{0.2}$ interface and the reduction of the GRL concentration.

In this section, we focus on the possible driving forces for such behaviors. We consider first the effect of strain, and then the minimization of the free energy of the system.

\section{Influence of strain}

The evolution in such systems is driven by energetic and entropic effects. The system's energy comes from the long-range elastic interactions and the short-range atomic interactions. We first show that the evolution described above is not due to the strain relaxation, which should favor conversely an homogeneous system. The elastic energy stored in sandwich-like geometry under study could be described by the strain sharing between the different layers, which would be the lowest strain state of our system. We consider a geometry relevant to the experimental systems under investigation, see Fig. 7. It consists of a buffer Si layer of thickness $e$, below the $\mathrm{Si}_{1-y} \mathrm{Ge}_{y}$ layer with the initial concentration $y=20 \%$ and the $\mathrm{Si}_{1-x} \mathrm{Ge}_{x}$ layer resulting from the oxidation process. We choose to describe generically the layer's lattice parameters $a$ with the linear approximation $a\left(S i_{1-x} G e_{x}\right)=x a(S i)+(1-x) a(G e)$. The upper (above $\mathrm{Si}_{1-x} \mathrm{Ge}_{x}$ ) and lower (below $\mathrm{Si}$ ) surfaces are supposed strain free as they are in contact with $\mathrm{SiO}_{2}$. The other interfaces are supposed coherent so that strain is shared between the different layers. For a planar system, the displacements at mechanical equilibrium may be solved exactly, ${ }^{31,32}$ and we find that the total elastic energy $E^{e l}=$ $e \mu^{S i}+h_{y} \mu^{y}+h_{x} \mu^{x}$ per unit surface (with the homogeneous elastic energy densities $\mu$ in the 
different layers) is

$$
E^{e l}=\frac{Y \Delta_{a}^{2}}{1-\nu} \frac{h_{x}\left(e+h_{y}\right) x^{2}-2 h_{x} h_{y} x y+h_{y}\left(e+h_{x}\right) y^{2}}{e+h_{x}+h_{y}},
$$

with the layers' Young's modulus $Y$ and Poisson's ratio $\nu$, and the SiGe misfit $\Delta_{a}=[a(G e)-$ $a(S i)] / a(S i)$.

The elastic energy depends on the different misfits ruled by $x$ and $y$, and on the different layers' thicknesses $e, h_{y}$ and $h_{x}$. In order to describe the experimental system, we choose $y$ constant, equal to its initial value $y=20 \%$. Moreover, as a first approximation, Ge may be considered as being out of the oxidation process so that mass conservation may be applied to this species : $x h_{x}+y h_{y}=y h_{y}^{0}$. In the first oxidation step, only the first SiGe layer is impacted by oxidation so that $h_{x}, h_{y}$ and $x$ may evolve, but not the underneath Si layer thickness $e$. We can consider that the only free parameters are $x$ and $h_{x}$. The oxidation process extracts Si atoms for the upper layer, so that $x$ is necessarily larger than the initial film concentration $(x \geq y)$. We plot in Fig. 8 the elastic energy as a function of $x$ and $h_{x}$. We see that for every $h_{x}$, the energy always increases with $x$ so that the homogeneous solution $x=y$ should always be preferred by the minimization of the elastic energy alone. More explicitly, one may compute the derivative of the elastic energy

$$
\left.\frac{\partial E^{e l}}{\partial x}\right)_{h_{y}}=\frac{y\left(h_{y}^{0}-h_{y}\right)\left[\left(e+h_{y}\right) x-y h_{y}\right]\left[e x+(x-y) h_{y}+2 h_{y}^{0} y\right]}{\left(e x+h_{y}(x-y)+y h_{y}^{0}\right)^{2}}
$$

which is always positive when $x \geq y$ so that $E^{e l}$ is indeed minimum for $x=y$. Hence, elasticity clearly favors an homogeneous solution and cannot explain the formation of the GRL. 


\section{High temperature regime}

We investigate here the effects of alloying, which involve both energetic and entropic contributions. We consider the same geometry as previously described with different layers. The top of the SiGe layer (thickness $h_{o x}$ ) is oxidized. The Ge atoms are repelled from oxygen and then diffuse among the monolayers (MLs) beneath. We consider that the diffusion front reaches the next MLs on a depth $h_{x}$, which assure conservation of Ge atoms. We do not impose here that the concentration in this GRL is $50 \%$. Si atoms are on the contrary not conserved as they may diffuse upward and be captured by $\mathrm{O}$ to form extra $\mathrm{SiO}_{2}$. We hence have a diffusion layer of thickness $h_{x}$, with a uniform Ge composition $x$ which arises from an initial layer of thickness $h_{o x}$ with the initial composition $y$. Ge conservation imposes

$$
h_{x} x=\left(h_{x}+h_{o x}\right) y \text {. }
$$

As regards the system's thermodynamics properties, we consider $N$ possible lattice sites with the probability $c$ of having a Ge atom (and thus, a probability $1-c$ for Si atoms). In a mean-field limit, the probability on the different lattice sites are independent from one another, and the mean energy $U$ and entropy $S$ of $N$ sites are

$$
\begin{aligned}
& U=\frac{1}{2} z N\left[\bar{\epsilon}+2 \delta \epsilon c(1-c)+2 \Delta \epsilon\left(c-\frac{1}{2}\right)\right] \\
& S=-N k_{B}[c \ln c+(1-c) \ln (1-c)]
\end{aligned}
$$

where $z$ is the crystal coordination number, $\bar{\epsilon}=\frac{1}{2}\left(\epsilon_{S i S i}+\epsilon_{G e G e}\right), \delta \epsilon=\epsilon_{S i G e}-\bar{\epsilon}$ and $\Delta \epsilon=$ $\frac{1}{2}\left(\epsilon_{G e G e}-\epsilon_{S i S i}\right)$, where $\epsilon_{A B}$ design the interaction between atoms $A$ and $B$. The formation free energy density ( $\left.f^{\text {form }}\right)$ which corresponds to the free energy difference between the initial $h_{o x}+h_{x}$ layers with a composition $y(i)$, and the final $h_{x}$ layers with a composition $x(f)$ is 
merely

$$
f^{\text {form }}=\frac{a}{N^{2 D}}\left(F^{f}-F^{i}\right)=h_{x}[f(x)-f(y)]+h_{o x}[\Delta \mu-f(y)]
$$

where $N^{2 D}$ is the number of lattice sites per layer, while

$$
\begin{aligned}
f(x)=z \delta \epsilon x(1-x)+z \Delta \epsilon\left(x-\frac{1}{2}\right) & \\
& +k_{B} T[x \ln (x)+(1-x) \ln (1-x)] .
\end{aligned}
$$

We define in (6) the driving force of oxidation, $\Delta \mu=\mu^{\mathrm{SiO}_{2}}-\frac{1}{2} z \bar{\epsilon}$, with the chemical potential $\mu^{\mathrm{SiO}_{2}}$ in the oxidized layer.

For a given oxidized thickness, i.e. for a given $h_{o x}$, we find that the minimum of free energy corresponds to the infinite dilution of the extra Ge coming from the oxidized layer (see Fig. 10). This situation corresponds to $h_{x} \rightarrow \infty$, with $x \rightarrow y$ due to Eq. (3). These results correspond to the experimental results described above in the case of high temperature where the complete oxidation leads to a dilute system compatible with mass conservation.

\section{Low temperature regime}

When the experiments are performed at low temperatures $\left(\leq 900^{\circ} \mathrm{C}\right)$, the GRL maintains a constant concentration $(\mathrm{x}=0.5)$. We have shown that the oxidation and the GRL growth follow a diffusive limited behavior (linear evolution with square root of time) with an activation energy lower than the diffusion of Ge through $\mathrm{Si}_{0.8} \mathrm{Ge}_{0.2}$. In this regime, the minimization of free energy of the whole system (Fig. 10) is kinetically inhibited due to the high diffusion barrier of $\mathrm{Ge}$ in $\mathrm{Si}_{0.8} \mathrm{Ge}_{0.2}$. In fact, at $750{ }^{\circ} \mathrm{C}$, the diffusion coefficient of $\mathrm{Ge}$ in $\mathrm{Si}_{0.5} \mathrm{Ge}_{0.5}$ is 5 orders of magnitude higher than in $\mathrm{Si}_{0.8} \mathrm{Ge}_{0.2}$. The Ge can thus be considered free to move inside the GRL and in that case, the excess of Ge at the top of the GRL due to oxidation of Si will diffuse towards the bottom of the layer to keep $\mathrm{x}=0.5$ inside the whole GRL. This $\mathrm{x}=0.5$ concentration is stabilized by the minimum entropy term of the $\mathrm{x}=0.5$ alloy (Fig. 9). 
At the bottom of the GRL, since there is almost no diffusion of Ge in $\mathrm{Si}_{0.8} \mathrm{Ge}_{0.2}$, we suggest that the Ge in excess is expeled from the GRL following a rapid local exchange mechanism similar to a two layers segregation mechanism. In this two layers system, the combination of kinetic frustration and local minimum of energy would then lead to the stabilisation of $\mathrm{x}=0.5$. Such a situation would explain the low activation energy of GRL formation and the diffusive-like behavior. The driving force of the segregation is the minimum of energy in the formation of the $\mathrm{Si}_{0.5} \mathrm{Ge}_{0.5}$ alloy.

To summarize, we demonstrated that the $50 \%$ Ge concentration observed in the low temperature regime is not the minimum of energy (elastic and free energy) of the system. We suggest that this concentration is metastable and controlled by a kinetic barrier which corresponds to the inhibition of the diffusion of $\mathrm{Ge}$ in $\mathrm{Si}_{0.8} \mathrm{Ge}_{0.2}$. However, a local exchange of Si and Ge atoms on two layers at the $\mathrm{Si}_{0.5} \mathrm{Ge}_{0.5} / \mathrm{Si}_{0.8} \mathrm{Ge}_{0.2}$ interface allows the Ge atoms from the $\mathrm{Si}_{0.5} \mathrm{Ge}_{0.5}$ GRL to reach the first monolayer of the $\mathrm{Si}_{0.8} \mathrm{Ge}_{0.2}$. At high temperature, the kinetic barrier becomes negligible, which explains the homogenization of the Ge concentration in the whole structure.

\section{Conclusions}

During the last years there was a huge interest for Ge on insulator layers for ultra-scales MOS transistors with innovative properties. The aim is to pursue the Moore's law by developing planar transistors based on ultra-thin Silicon Germanium on insulator (UTSGOI) systems. One major hurdle is the fabrication process of the strained Silicon-Germanium (or

pure Germanium) layers on insulator (SSGOI). While UTSGOI are already used as vehicle tests for the next generation of transistors (node 14nm), the formation of the Ge rich layer by condensation during SiGe thermal oxidation and the stability/homogenization of the concentration are still controversial.

We use here two systems with different initial strain and we demonstrate two fabrica- 


\section{Acknowledgement}

The authors thank Alain Portavoce for fruitfull discussions.

\section{References}

(1) Suh, J.; Nakane, R.; Taoka, N.; Takenaka, M.; Takagi, S. Highly Strained-SiGe-OnInsulator P-Channel Metal-Oxide-Semiconductor Field-Effective Transistors Fabricated 
by Applying Ge Condensation Technique to Strained-Si-On-Insulator Substrates. Appl. Phys. Lett. 2011, 99, 142108.

(2) Liu, X.; Ma, X.; Du, X.; Liu, W.; Song, Z.; Lin, C. Modified Postannealing of the Ge Condensation Process for Better-Strained Si Material and Devices. J. Vac. Sci. \& Technol. B 2010, 28, 1020-1025.

(3) Smith, C. E.; Adhikari, H.; Lee, S.-H.; Coss, B.; Parthasarathy, S.; Young, C.; Sassman, B.; Cruz, M.; Hobbs, C.; Majhi, P. et al. Dual Channel FinFETs As a Single High-K/metal Gate Solution Beyond 22nm Node. Electron Devices Meet. (IEDM), 2009 IEEE Int. 2009; pp 1-4.

(4) Jiang, Y.; Singh, N.; Liow, T. Y.; Lo, G. Q.; Chan, D. S. H.; Kwong, D. L. Reduced Carrier Backscattering in Heterojunction SiGe Nanowire Channels. Appl. Phys. Lett. 2008, 93, 253105.

(5) Tezuka, T.; Nakaharai, S.; Moriyama, Y.; Sugiyama, N.; Takagi, S. High-Mobility Strained SiGe-On-Insulator PMOSFETs with Ge-Rich Surface Channels Fabricated by Local Condensation Technique. Electron Device Lett. IEEE 2005, 26, 243-245.

(6) Jain, J. R.; Hryciw, A.; Baer, T. M.; Miller, D. A. B.; Brongersma, M. L.; Howe, R. T. A Micromachining-Based Technology for Enhancing Germanium Light Emission Via Tensile Strain. Nat. Photonics 2012, 6, 398-405.

(7) Tezuka, T.; Sugiyama, N.; Mizuno, T.; Suzuki, M.; Takagi, S.-i. A Novel Fabrication Technique of Ultrathin and Relaxed SiGe Buffer Layers with High Ge Fraction for Sub-100nm Strained Silicon-On-Insulator MOSFETs. Jpn. J. Appl. Phys. 2001, 40, $2866-2874$.

(8) Boyd, I. W.; Craciun, V.; Kazor, A. Vacuum-Ultra-Violet and Ozone Induced Oxidation of Silicon and Silicon-Germanium. Jpn. J. Appl. Phys. 1993, 32, 6141. 
(9) Madsen, J. M.; Cui, Z.; Takoudis, C. G. Low Temperature Oxidation of SiGe in Ozone: Ultrathin Oxides. J. Appl. Phys. 2000, 87, 2046-2051.

(10) Lim, Y. S.; Jeong, J. S.; Lee, J. Y.; Kim, H. S.; Shon, H. K.; Kim, H. K.; Moon, D. W. A Study of the Oxidation Behavior and the Postannealing Effect in a Graded SiGe/Si Heterostructure. J. Electron. Mater. 2002, 31, 529-534.

(11) Riley, L. S.; Hall, S. X-Ray Photoelectron Spectra of Low Temperature Plasma Anodized Si0.84Ge0.16 Alloy on Si(100): Implications for SiGe Oxidation Kinetics and Oxide Electrical Properties. J. Appl. Phys. 1999, 85, 6828-6837.

(12) Xie, Y. H. SiGe Field Effect Transistors. Mater. Sci. Eng. R Reports 1999, 25, 89-121.

(13) Nakaharai, S.; Tezuka, T.; Sugiyama, N.; Moriyama, Y.; Takagi, S.-i. Characterization of 7-Nm-Thick Strained Ge-On-Insulator Layer Fabricated by Ge-Condensation Technique. Appl. Phys. Lett. 2003, 83, 3516-3518.

(14) Xue, Z. Y.; Di, Z. F.; Ye, L.; Mu, Z. Q.; Chen, D.; Wei, X.; Zhang, M.; Wang, X. Study of Ge Loss During Ge Condensation Process. Thin Solid Films 2014, 557, 120-124.

(15) Fathy, D.; Holland, O. W.; White, C. W. Formation of Epitaxial Layers of Ge on Si Substrates by Ge Implantation and Oxidation. Appl. Phys. Lett. 1987, 51, 1337-1339.

(16) Pizzini, S.; Acciarri, M.; Binetti, S.; LeDonne, A.; Marchionna, S.; Bollani, M. Defect Studies on Silicon and Silicon-Germanium for PV and Optoelectronic Applications. Mater. Sci. Semicond. Process. 2006, 9, 66-73.

(17) Kanoun, M.; Souifi, A.; Decossas, S.; Dubois, C.; Bremond, G.; Bassani, F.; Lim, Y.; Ronda, A.; Berbezier, I.; Kermarrec, O. et al. Oxidation of Si / Nc-Ge / Si Heterostructures for Non Volatile Memory Applications. Symp. Q: Unconv. Approaches to Nanostructures with Appl. Electron. Photonics, Inf. Storage Sens. 2003. 
(18) Long, E.; Galeckas, A.; Yu Kuznetsov, A.; Ronda, A.; Favre, L.; Berbezier, I.; Radamson, H. H. Nano-Structuring in SiGe by Oxidation Induced Anisotropic Ge SelfOrganization. J. Appl. Phys. 2013, 113, 104310.

(19) Sugiyama, N.; Tezuka, T.; Mizuno, T.; Suzuki, M.; Ishikawa, Y.; Shibata, N.; Takagi, S. Temperature Effects on Ge Condensation by Thermal Oxidation of SiGe-On-Insulator Structures. J. Appl. Phys. 2004, 95, 4007-4011.

(20) Balakumar, S.; Lo, G. Q.; Tung, C. H.; Kumar, R.; Balasubramanian, N.; Kwong, D. L.; Ong, C. S.; Li, M. F. SiGe Amorphization During Ge Condensation in Silicon Germanium on Insulator. Appl. Phys. Lett. 2006, 89, 42115.

(21) Wang, G. H.; Toh, E.-H.; Foo, Y.-L.; Tung, C.-H.; Choy, S.-F.; Samudra, G.; Yeo, Y.C. High Quality Silicon-Germanium-On-Insulator Wafers Fabricated Using Cyclical Thermal Oxidation and Annealing. Appl. Phys. Lett. 2006, 89, 53109.

(22) Nguyen, Q. T.; Damlencourt, J. F.; Vincent, B.; Clavelier, L.; Morand, Y.; Gentil, P.; Cristoloveanu, S. High Quality Germanium-On-Insulator Wafers with Excellent Hole Mobility. Solid. State. Electron. 2007, 51, 1172-1179.

(23) Min, B.-G.; Pae, Y. H.; Jun, K. S.; Ko, D.-H.; Kim, H.; Cho, M.-H.; Lee, T.-W. Formation of a Ge-Rich Layer During the Oxidation of Strained $S i_{1-x} G e_{x}$. J. Appl. Phys. 2006, 100, 16102.

(24) Ma, X.; Liu, W.; Chen, C.; Du, X.; Liu, X.; Song, Z.; Lin, C. Fabrication of High Ge Content SiGe-On-Insulator with Low Dislocation Density by Modified Ge Condensation. Appl. Surf. Sci. 2009, 255, 7743-7748.

(25) Sugiyama, N.; Nakaharai, S.; Hirashita, N.; Tezuka, T.; Moriyama, Y.; Usuda, K.; Takagi, S. The Formation of SGOI Structures with Low Dislocation Density by a TwoStep Oxidation and Condensation Method. Semicond. Sci. Technol. 2007, 22, S59. 
(26) Pichler, P. Intrinsic Point Defects, Impurities, and Their Diffusion in Silicon; SpringerVerlag: Wien New York - Austria, 2004.

(27) Gusakov, V. Unified Model of Diffusion of Interstitial Oxygen in Silicon and Germanium Crystals. J. Phys. Condens. Matter 2005, 17, S2285.

(28) Stolwijk, N. A.; Bracht, H. Landolt-Bornstein-Numerical Data and Functional Relationships in Science and Technology; D. Beke Springer-Verlag: Berlin, 1998; Vol. III-33A.

(29) Glowacki, F.; Royer, C. L.; Morand, Y.; Pédini, J.-M.; Denneulin, T.; Cooper, D.; Barnes, J.-P.; Nguyen, P.; Rouchon, D.; Hartmann, J.-M. et al. Ultrathin (5 Nm) SiGe-On-Insulator with High Compressive Strain (>2 GPa): From Fabrication (Ge Enrichment Process) to In-Depth Characterizations. Solid. State. Electron. 2014, 97, $82-87$.

(30) Vincent, B.; Damlencourt, J.-F.; Delaye, V.; Gassilloud, R.; Clavelier, L.; Morand, Y. Stacking Fault Generation During Relaxation of Silicon Germanium on Insulator Layers Obtained by the Ge Condensation Technique. Appl. Phys. Lett. 2007, 90, 74101.

(31) Xu, X.; Aqua, J.-N.; Frisch, T. Growth Kinetics in a Strained Crystal Film on a Wavy Patterned Substrate. J. Phys. Condens. Matter 2012, 24, 45002.

(32) Gaillard, P.; Aqua, J.-N.; Frisch, T. Kinetic Monte Carlo Simulations of the Growth of Silicon Germanium Pyramids. Phys. Rev. B 2013, 87, 125310. 
a Depth profiles of Ge concentration as a function of oxidation time:
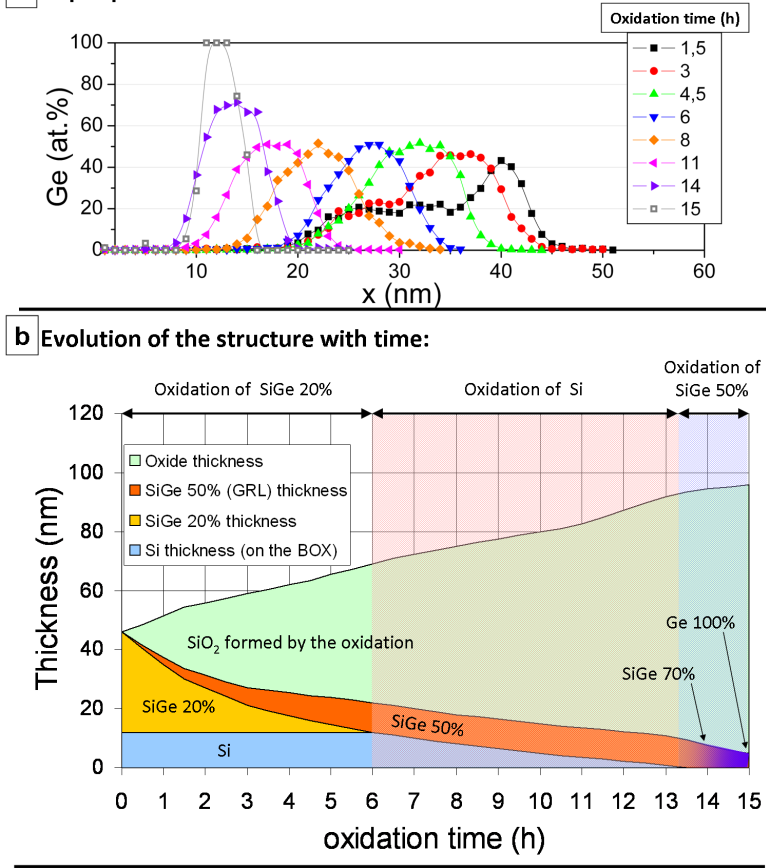

c Dark field STEM images as a function of oxidation time:
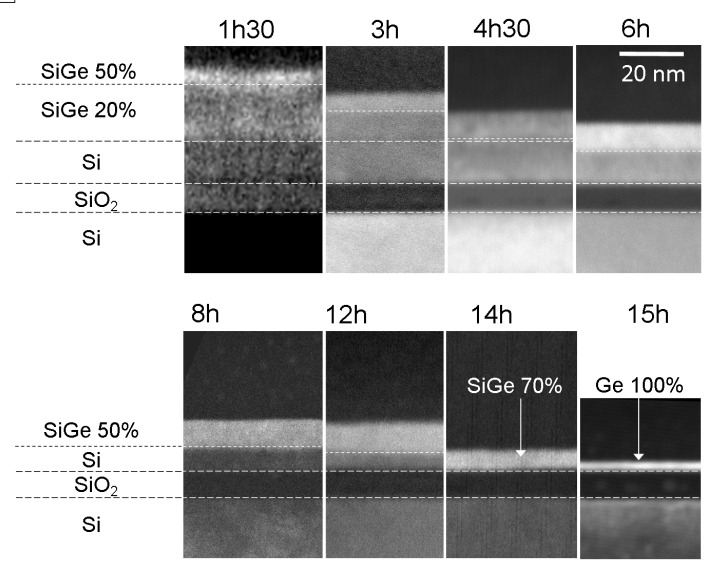

Figure 5: (a) Germanium concentration profiles measured by STEM-EDS analysis on cross sections of the $\mathrm{Si}_{0.8} \mathrm{Ge}_{0.2}$ /UTSOI structure after oxidation at $750{ }^{\circ} \mathrm{C}$ for different durations. (b) Schematic view of the evolution of the structure for reference. (c) Corresponding darkfield STEM cross-section images, all on the same scale. 


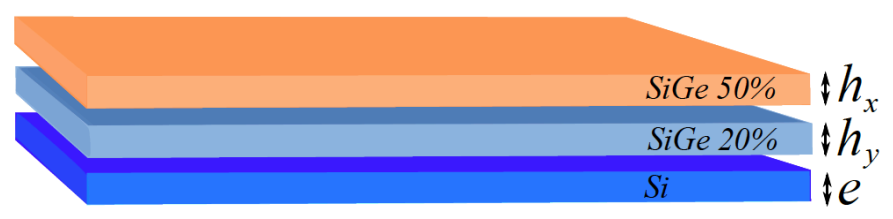

Figure 7: Geometry under investigation in the modelization.

Figure 6: (a) HRTEM image and (b) STEM-HAADF image of the structure after $8 \mathrm{~h}$ of $750{ }^{\circ} \mathrm{C}$ oxidation: $10 \mathrm{~nm}$ thick $\mathrm{Si}_{0.5} \mathrm{Ge}_{0.5}$ layer on $7 \mathrm{~nm}$ thick UTSOI. Thicknesses of the layers match the EDS profile of figure 5 very well.

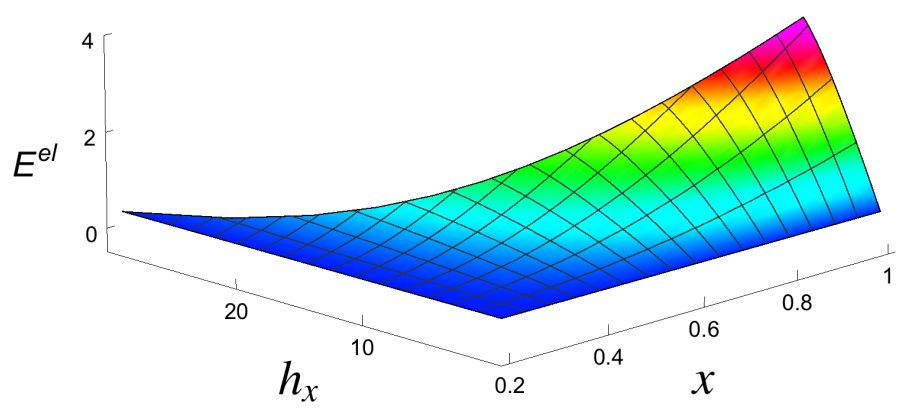

Figure 8: Elastic energy (in arbitrary units) as a function of the top layer concentration $x$ and thickness $h_{x}$, in the authorized region where $x \geq y$ and where $h_{y}$, dictated by the Ge mass conservation, satisfies $h_{y} \geq 0$. 


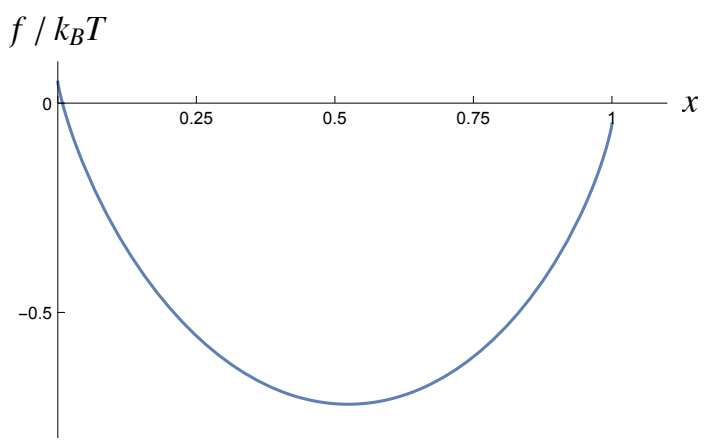

Figure 9: Free energy $f$ of a layer with $\delta \epsilon<0$ and $\Delta \epsilon$ small either compared either to $\delta \epsilon$ or $k_{B} T$, which is a typical case for $\mathrm{Si} / \mathrm{Ge}$ systems which is a solid solution. As a consequence, $f$ is minimum at $x=0.5$.

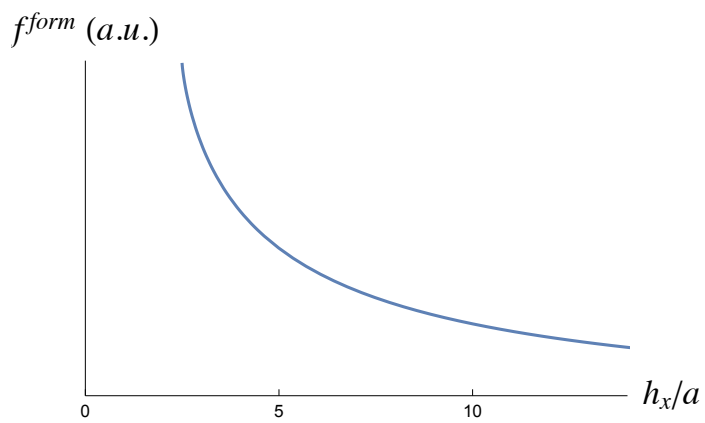

Figure 10: Formation free energy (6) (in arbitrary units) of a system with a given thickness $h_{o x}$ of oxidized layers and a diffusion layer of thickness $h_{x}$ with a mean Ge concentration satisfying the Ge conservation (3).

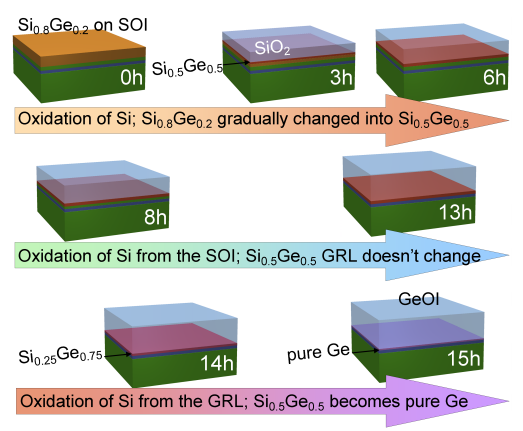

Figure 11: Graphical TOC 